Ann. Biol. anim. Bioch. Biophys., I970, 10 (4), 575-579.

\title{
ÉTUDE SUR LE NOMBRE D'OVULATIONS OBTENUES APRES INJECTION DE PMSG CHEZ LA VACHE : NOTION DE SEUIL DE RÉPONSE
}

\author{
J.-C. MARIANA \\ Station de Recherches sur la Physiologie de la Reproduction, \\ Centre de Recherches de Tours, 37 - Nouzilly \\ Institut national de la Recherche agronomique \\ RÉSUMÉ
}

L'hétérogénéité de la réponse des ovaires de vache : nombre d'ovulations à des doses croissantes de PMSG nou's a conduit à envisager un modèle de réponse par scuils et à calculer les seuils correspondant à $\mathrm{I}, 2,3,4$ ovulations ainsi que l'écart type des seuils.

La réponse : nombre d'ovulations obtenues à la suite d'un traitement destiné à induire une superovulation chez des vaches, est d'une part essentiellement discontinue, d'autre part extrêmement variable.

En effet, pour une même dose d'hormone injectée, la réponse peut varier dans des proportions considérables suivant les conditions de traitement et l'état physiologique de l'animal.

Par ailleurs, même pour des doses élevées d'hormone, la proportion de réponses représentées par une seule ovulation reste importante (Mariana et al., I970). Dans cette espèce, il n'a pas été possible, jusqu'à présent, de mettre en évidence une relation linéaire entre la dose d'hormone injectée et le nombre d'ovulations obtenues.

Certains auteurs ont examiné la possibilité d'une telle relation linéaire chez d'autres espèces. Citons Robinson (I95I) et BAKER et CoGGIns (Ig68). Mais l'hétérogénéité de la variance entre doses reste très importante chez la Brebis ; cette hétérogénéité doit avoir une influence sur la possibilité d'étude d'une relation linéaire. Chez la Truie, cependant, il semble qu'on ait un meilleur ajustement entre doses et nombre d'ovtulations. On peut remarquer que, chez la Truie, le nombre d'ovulations. est naturellement élevé. 
L,e problème est donc peut-être un problème d'espèce. Les mécanismes présidant à la maturation folliculaire sont différents d'une espèce à l'autre, en particulier pour ce qui concerne le rendement de la maturation folliculaire (MARION et GIER, Ig68).

Pour expliquer la variabilité du nombre d'ovulations après traitement par PMSG chez la Vache, nous avons envisagé la possibilité d'une réponse par seuils : pour un animal donné, le nombre d'ovulations provoquées à la suite d'un traitement, restera constant jusqu'à une dose telle qu'au-delà la réponse sera accrue ; cette dose pourra être appelée seuil de réponse $S_{i}$.

Pour cet anima1, on peut envisager que sa réponse au traitement se développe selon le schéma suivant (fig. I).

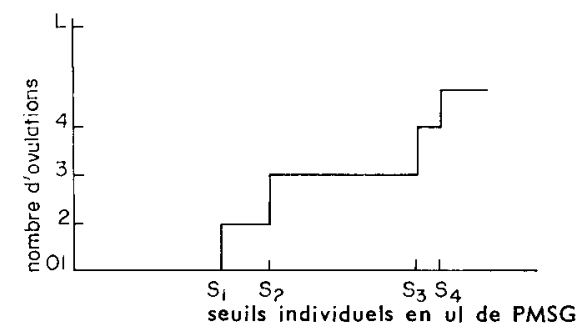

FIG I - Représentation des réponses au niveau de l'individu

Les valeurs $S_{1}, S_{2}, \ldots, S_{n-1}$ de dose PMSG qui permettent d'accroittre la réponse d'une unité peuvent être considérées comme les seuils successifs de réponse au traitement. En fait, les animaux ont des sensibilités différentes. On supposera, à cet égard, que la sensibilité d'une population nombreuse exprimée en dose d'hor-

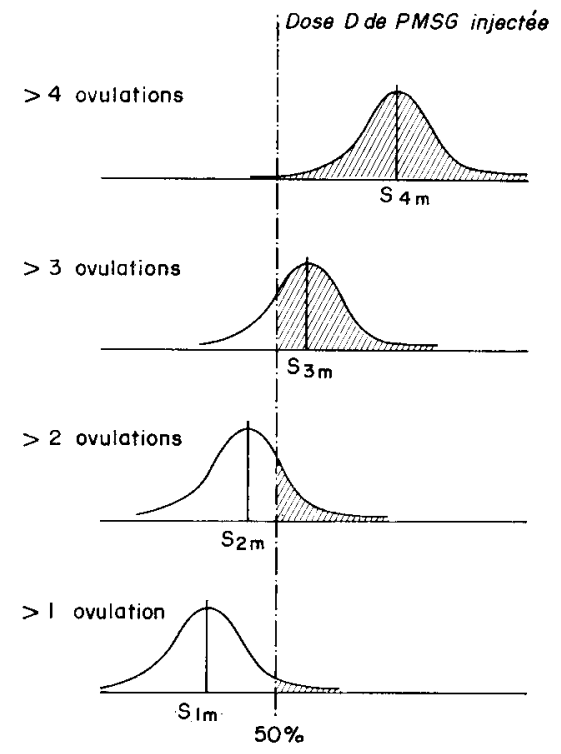

FIG. 2. - Schéma théorique de réponse: nombre d'ovulations des ovaires de vache $\dot{a}$ des doses croissantes de PMSG 
mone obéit à une loi normale. Ainsi, les valeurs $S_{1}, S_{2}, \ldots, S_{n_{-1}}$ sont distribuées autour de valeurs moyennes $S_{1 m}, S_{2}, \ldots, S_{(n-1) m}$ avec le même écart type (fig. 2). Avec cette hypothèse, la dose injectée $D_{i}$ apparaît comme la valeur moyenne des seuils qu'elle découvre.

Sur la figure 2 bis, on lit directement la proportion d'animaux répondant par $i$ ovulations au traitement $\mathrm{D}_{i}$.

\begin{tabular}{c|c|c}
\hline $\begin{array}{c}\text { Proportions d'animaux } \\
\text { pour lesquels le nombre } \\
\text { d'ovulations est }\end{array}$ & \multicolumn{2}{|c}{ Doses } \\
\cline { 2 - 3 } & $\mathrm{D}_{1}$ & $\mathrm{D}_{2}$ \\
\hline 1 ou 2 & $\mathrm{P}_{11}$ & $\mathrm{P}_{\mathbf{2 1}}$ \\
\hline $\mathrm{P}_{\mathbf{1 2}}$ & $\mathrm{P}_{\mathbf{2 2}}$ \\
\hline
\end{tabular}

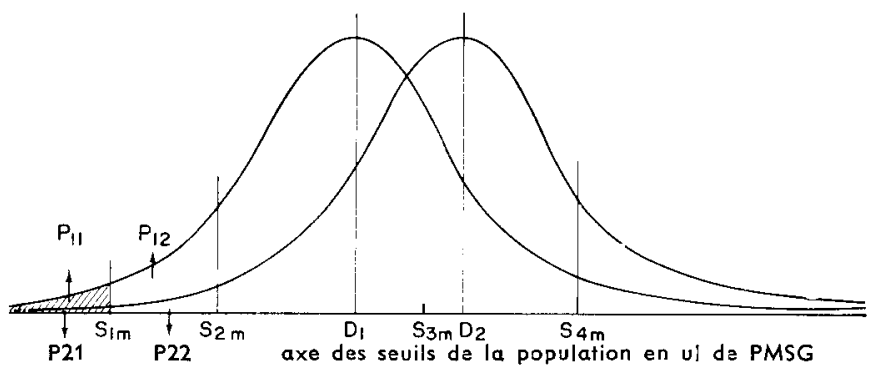

FlG. 3. - Schéma d'évolution des pourcentages de réponses à une $D_{i}$ dose de PMSG.

(L'écart type $\sigma$ est stupposé indépendant de la dose)

D'une façon générale $\mathrm{P}_{i j}$ est la proportion d'animaux ayant eu jusqu'à $j$ orulations au traitement $i$

$$
(i=\mathrm{I} .2 \ldots k, j=\mathrm{I} .2 \ldots \ldots n-\mathrm{I}) .
$$

Soit $\mathrm{U}_{i j}$ la valeur de la variable normale réduite telle que :

$$
\mathrm{F}\left(\mathrm{U}_{i j}\right)=\mathrm{P}_{i j} ; \mathrm{U}_{i j}=\frac{\mathrm{S}_{j m}-\mathrm{D}_{i}}{\sigma}
$$

On connaît des estimations des $U_{i j}$ à partir des proportions observées correspondant aux proportions théoriques $\mathrm{P}_{i j}$.

L'estimation des $S_{J m}$ et de $\sigma$ peut être obtenue par la méthode des moindres carrés, à partir de 1'équation :

$$
\frac{\mathrm{S}_{\mathrm{J} m}-\mathrm{D}_{i}}{\sigma}-\mathrm{U}_{i j}=\mathrm{o}
$$

Il s'agit de trouver les estimations $\widehat{\mathrm{S}}_{j m}$ et $\hat{\sigma}$ qui minimisent l'expression :

$$
\sum_{i}\left(\frac{\mathrm{S}_{j}-\mathrm{D}_{i}}{\sigma}-\mathrm{U}_{i j}\right)^{2}
$$


On trouve $\hat{\mathrm{S}}_{\mathrm{J} m}=\overline{\mathrm{D}}+\sigma \mathrm{U}_{o j}$ en posant :

$$
\begin{gathered}
\overline{\mathrm{D}}=\frac{\Sigma \mathrm{D}_{i}}{\mathrm{~K}} \text { et } \mathrm{U}_{o j}=\frac{\Sigma \mathrm{U}_{i j}}{\mathrm{~K}} \\
\text { et } \hat{\sigma}=\frac{\Sigma\left(\mathrm{D}_{i}-\overline{\mathrm{D}}\right)^{2}}{\Sigma \mathrm{U}_{i o}\left(\overline{\mathrm{D}}-\mathrm{D}_{i}\right)} \quad \text { en posant } \mathrm{U}_{i o}=\frac{\Sigma \mathrm{U}_{i j}}{n-\mathrm{I}}
\end{gathered}
$$

\begin{tabular}{c|r|r|r}
\hline \hline \multirow{2}{*}{ Nombre d'ovulations } & \multicolumn{3}{|c}{ Doses } \\
\cline { 2 - 4 } & 2400 U.I. & 3000 U.I. & 3750 U.I. \\
\hline 1 & 12 & 12 & 14 \\
2 & 6 & 4 & 3 \\
3 & 3 & 1 & 1 \\
4 & 4 & 4 & 1 \\
5 & 1 & 1 & 2 \\
\hline$>6$ & 3 & 8 & 3 \\
\hline Total en animaux & 30 & 30 & 30 \\
\hline \hline
\end{tabular}

La théorie précédente nous donne pour les 6 premiers seuils et l'écart type, les valeurs suivantes en unités internationales de PMSG.

$$
\begin{aligned}
\mathrm{S}_{1}=224 \mathrm{I} & \mathrm{S}_{2}=374 \mathrm{I} \\
\mathrm{S}_{3}=4346 & \mathrm{~S}_{4}=5557 \\
\mathrm{~S}_{5}=6 \text { I } 44 & \mathrm{~S}_{6}=6509 \\
\text { et } \sigma=4 \text { IIO } &
\end{aligned}
$$

Ces estimations sont assez grossières étant donné le faible nombre d'animaux. On remarquera également la grande hétérogénéité des animaux quant à l'action de l'hormone.

\section{DISCUSSION}

La notion de " sensibilité " (" liability ") sous-jacente à un seuil a été développée de façon quantitative par le généticien FALCONER en I965, à propos de l'étude de l'hérédité de certaines maladies (maladie de la peau, ulcère peptique) de type tout ou rien.

Dans la mesure où ce modèle a quelque vraisemblance, on ne doit pas s'attendre à obtenir des résultats homogènes par un traitement de superovulation. Ce modèle suppose que la distribution des seuils reste normale et de même écart type sur toute l'étendue des doses : de ce fait, il est nécessaire que le mécanisme physiologique reste homogène. C'est peut-être inexact pour les fortes doses (McLAREN, Ig66). L'écart type augmente vraisemblablement avec la dose. On peut cependant penser que la dose de PMSG injectée agit au niveat de l'ovaire en préparant un stock de follicules ou en maturant plus de follicules que normalement, mais que seulement une certaine 
proportion des follicules ovuleront ; le problème se situe alors au niveau de l'ovulation et de son contrôle par l'hypophyse de l'animal.

Si le modèle proposé ne rejette pas le fait que la variance du nombre d'ovulations augmente avec les doses injectées, il ne l'explique pas cependant.

\title{
REMERCIEMENTS
}

Nous tenons à remercier M. Vessereau. La présente étude a bénéficié de ses nombreux conseils dans la mise en forme.

\author{
SUMMARY \\ INVESTIGATION ON OVULATION AFTER PMSG INJECTION \\ IN THE COW. THRESHOLD OVULATION NUMBERS
}

The heterogeneity of the number of eggs shed by the cow in response to increasing doses of PMSG led to the development of a model to represent the variation as a continuous variable.

The mean thresholds between $\mathbf{I}, 2,3,4$ and 5 ovulations were calculated together with their standard deviations.

\section{RÉFÉRENCES BIBLIOGRAPHIQUES}

BAKER R. A., CogGins E. G., r968. Control of ovulation rate and fertilization in prepuberal gilts. $J$. Anim. Sici., 2\%, I607-I610.

Falconer I). S., I965. The inheritance of liability to certain diseases estimated from the incidence among relatives. Ann. Hum. Genet. London, 29, 5 I-76.

FALCONER I).S., 1967 . The inheritance of liability to diseases with variable age of onset with particular reference to diabetes mellitus. Ann. Hum. Genet. London., 31, I-20.

McLaren A., 1966. Regrulation of ovulation rate after removal of one ovary in mice. Proc. roy. Soc. B., 166, 3i $6-3+0$.

Mariana J.-C., Mauléon P., Benott M., Chupix D., I970. Variabilité et répétabilité du nombre d'ovulations obtenues apres injection de I 600 UI de PXSG et de I 500 UI de HCG. Ann. Biol. anim. Bioch. Biophys., 10, no hors série $\mathrm{x}, 47-63$.

Marion G. B., Gier H. T., Chovdary J. B., i969. Micromorphology of the bovine ovarian follicular system. J. Anim. Sci, 2\%, 45I-65.

Robinsov T. J., 195I. The control of fertility in sheep. Part. II. The augmentation of fertility by gonadotrophin treatment of the ewe in the normal breeding season. J. A gric. Sci., 41, 6-63. 